\title{
Ventriloquism, sensory interaction, and response bias: Remarks on the paper by Choe, Welch, Gilford, and Juola
}

\author{
PAUL BERTELSON and MONIQUE RADEAU \\ Université libre de Bruxelles, 1050 Brussels, Belgium
}

\begin{abstract}
The arguments on which Choe, Welch, Gilford, and Juola have based their thesis that the various manifestations of ventriloquism are postperceptual effects are critically examined. The application of detection theory to the results of a discrepancy detection experiment, which they claimed allowed them to separate sensory interaction from response bias, is found invalid. It is further argued that an interpretation of ventriloquism exclusively in terms of response processes cannot account (1) for the compelling quality of the effect, and (2) for the occurrence of aftereffects.
\end{abstract}

Many everyday examples, including the trick performed by the ventriloquist, suggest that we have a remarkable ability to ignore a spatial discordance between auditory and visual evidence which can be related to a single event. The phenomenon which has come to be called ventriloquism deserves attention for a variety of reasons, one of which is that, unlike the more widely studied case of visuo-proprioceptive rearrangement, auditory-visual discrepancy is a situation where both inputs are under experimental control. The demonstration that exposure to auditoryvisual conflict produces aftereffects has brought further suggestion that we are dealing with an interesting case of perceptual plasticity.

In their recent paper, Choe, Welch, Gilford, and Juola (1975) suggest that this interest may be misplaced: the various effects of ventriloquism, they argue, are not perceptual events but, rather, manifestations of particular response tendencies. The argument is based mainly on the application of detection theory to the results of an experiment where subjects had to detect auditory-visual discrepancies. Ventriloquism was found to correspond to a change in the decision criterion measure, $\beta$, not in the sensitivity measure, $d^{\prime}$. In that experiment, where discrepancies in opposite directions were produced in random order, only one aspect of ventriloquismthe immediate interaction between simultaneously presented auditory and visual information, could be observed. The cumulative adaptive shifts in apparent location, which manifest themselves in aftereffects, cannot be studied in such a situation. The authors

This work was carried out while the second author held a grant from the Belgian "Fonds national de la Recherche scientifique." Thanks are due to André Ducamp, Jean-Claude Falmagne, Jose Morais, and Henri Rouanet for critical comments on a first version of the paper. The authors' address: Laboratoire de Psychologie expérimentale, 117 av. Adolphe Buyl, 1050 Bruxelles, Belgium. suggest that these shifts can be explained in terms of response learning.

A study where a fashionable analytical tool is applied to a traditional problem is likely to draw much attention. Choe et al.'s paper might have a great influence on work not only on ventriloquism, but on intermodal interactions more generally. It is thus necessary to examine carefully the various claims made by the authors.

\section{The Application of Detection Theory}

Detection theory is applied to the situation where a subject is exposed to spatially discordant auditory and visual information and has to say if the two signals come from the same location or not. The authors' remark that if one particular manipulationin their case, synchronization of auditory and visual events-increases the frequency of the response "same," the fact does not necessarily reflect a change in apparent location, and might also be due to an increased tendency to give that response, in spite of contradictory sensory evidence. In other words, the problem is to know if the interaction occurs before or after formation of a conscious impression of location.

In the experiment, the subjects are, on each trial, presented with a flash and a sound, coming either in the same place or in two locations $11^{\circ}$ apart horizontally and occurring either simultaneously or with various time intervals. For each timing condition, proportions of "same" responses on "discrepancy" (D) trials and on "no-discrepancy" (ND) trials are converted into the standard detection theory measures, $d^{\prime}$ and $\beta$. Synchronization is found to affect $\beta$, not $d^{\prime}$, which at first sight seems to support the authors' thesis that ventriloquism is a response bias phenomenon.

To apply detection theory, the authors assume that a subjective distance, $\mathrm{x}$, between sound and 


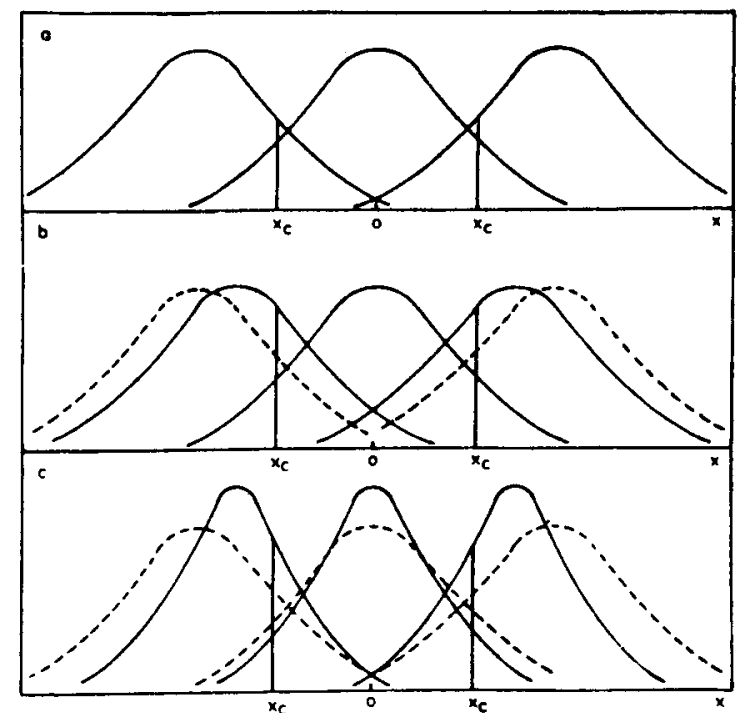

Figure 1. Statistical decision model: probability distributions of the apparent distances between tone and light for (from left to right) tone-left trials, no-discrepancy, and tone-right trials: (a) with asynchronous inputs ( $d^{\prime}$ and $x_{c}$ have been chosen to correspond to the mean probability values for condition $T / L-2.0$ of Choe et al.); (b) heavy lines-Choe et al.'s prediction for the case of sensory interaction, dotted lines-situation before interaction [cf.(a)]; (c) heavy lines-our prediction for sensory interaction consisting of a reduction of each apparent distance by a same percentage, dotted lines-before interaction.

light is available on each trial, and that the probability distributions of $\mathrm{x}$ for each objective sound-light distance $\left(11^{\circ}\right.$ left, $0^{\circ}, 11^{\circ}$ right $)$, under each timing condition, are known (Figure 1a). The subject chooses a criterion, $x_{c}$, for each timing condition and responds "same" whenever $x<x_{c}$. The way the two hypothetical mechanisms-sensory interaction and response bias-affect the parameters of the model is not discussed explicitly, and this is the point that needs to be examined.

For response bias, the translation is straightforward: the criterion, $x_{c}$, is shifted to higher values, hence $\beta$ decreases (if $\beta$ is expressed in terms of the likelihood ratio of the hypothesis "same"). For sensory interaction, the prediction is not so obvious. When they equate sensory interaction with a reduction in $\mathrm{d}^{\prime}$, the authors are apparently assuming that the probability distributions shift along the abscissa and increase their overlap (Figure 1b). ${ }^{1}$ This is, in fact, how a decrease in stimulus intensity is interpreted in standard applications of detection theory. In the present case, such horizontal translation could occur if, for example, the objective spatial discrepancy was reduced. Auditory-visual sensory interaction, however, can be expected to produce a very different type of transformation, where each subjective distance is reduced, whether it belongs to the ND distribution or to one of the $\mathrm{D}$ distributions. The result is a distortion of the abscissa scale. If, for instance, the rule is that each subjective distance is divided by a constant, the change will be as represented in Figure 1c: all three distributions now have a smaller variance. If, as seems more probable, small subjective distances are relatively more affected than larger ones, the variance of the ND distribution will be reduced more than those of the two $D$ distributions.

The important point is that this kind of transformation does not affect the alignment of the three distributions: after interaction has taken place, the area under the ND distribution which corresponds to the apparent distance with a given area under one $\mathrm{D}$ distribution is still the same. In other words, the ROC curve is not affected by sensory interaction. Hence, measures of discriminability which are based on the location of the ROC curve-like area under the curve or $d^{\prime}$, if conditions for computing it are fulfilled-will not change. On the other hand, the likelihood ratio corresponding to a particular subjective distance, $x$, will necessarily be affected; hence, if the subject keeps his criterion constant across the various timing conditions, $\beta$ will be affected.

It thus appears that the experiment run by the authors did not make it possible to measure sensory interaction as a change in detectability. It seems that it would be very difficult to find a design which provides a noncontroversial test. If sensory interaction is to affect $\mathrm{d}^{\prime}$, or any other measure of discriminability, we must compare responses in two groups of trials, one group affected by the interaction, the other not. For instance, compare responses on discrepancy trials with synchronous inputs and with asynchronous ones. But, since in such a case the trials can be discriminated on the basis of asynchrony itself, a positive result would become ambiguous, for the subjects might have used different decision criteria on the different trials.

\section{Ventriloquism as a Response Process}

The fact that the argument based on detection theory was invalid does not automatically eliminate Choe et al.'s suggestion that ventriloquism is reducible to the adoption of a particular response bias.

Before discussing that point, it is necessary to specify what is meant by response bias. The kind of criterion setting operation which is formalized in detection theory can occur at different depth levels. A bias can in consequence be established in favor of a particular perceptual interpretation, of a particular conclusion in a conscious inference process, or of a particular response. If response bias is used to include all these cases, there is little to disagree with, but the concept loses most of its usefulness. In particular, it is no more an alternative to sensory interaction.

That a bias towards an interpretation is compatible with sensory interaction, in the sense used by Choe 
et al. of an effect on apparent location, is important and must be insisted on. We can, for instance, imagine the following story. Sensory data about spatial position of, respectively, auditory and visual targets are compared to obtain a decision of same vs. different sources. The decision criterion is set on the basis of other relevant evidence derived from instructions, the visible context and the timing of the signals. On the other hand, the same data are translated into apparent locations. When the decision "same origin" is made and the existing translation rule would give discordant apparent locations, the rule is recalibrated until the discordance falls under some threshold value. This process is sensory interaction. When the decision is "different origin," no recalibration occurs. What reaches consciousness, and controls eventual localization responses, is the result of the translation process, not the data used for the same-or different-origin decision. We do not contend that this theory can be justified in all its details on the basis of existing data. ${ }^{2}$ Its interest is only to show that a reasonable perceptual account of ventriloquism can integrate the principle of a criterion shift.

If "response bias" is considered as an alternative to sensory interaction, the concept must thus be used in the more restricted sense of a conscious or postperceptual adjustment. Can ventriloquism be accounted for entirely in terms of such factors? It seems there are two categories of data which cannot be dealt with in that way.

(a) There is first the phenomenal evidence. The distinction between a perceptual interpretation and a conscious inference is after all a phenomenal one, and one cannot deal with it if one is not prepared to accept some kind of introspective data. Those who have been placed in the kind of experimental situation which Choe et al. are using can testify that the impression of fusion of the objectively discordant sources is a very compelling one, even with minimal contextual information. Thurlow and Jack (1973) have systematically recorded reports of apparent fusion, and have explored their variations with conditions of spatial separation and contextual suggestion. Those who insist that ventriloquism results from conscious rejection of part of the evidence would have to account for such variations. They would also have to explain why people pay to watch a ventriloquist's show.

(b) There is also the occurrence of aftereffects, which in adaptation research has generally been accepted as evidence for a perceptual recalibration, as opposed to a conscious correction. Choe et al. argue that aftereffects can be attributed to the learning of new responses during exposure to conflict. This suggestion, it must be noted, is different from the response bias explanation which was applied to the immediate effect of conflict, although it shares with it the insistance on nonperceptual factors. The authors base their discussion on part of Canon's $(1970,1971)$ findings, those concerning auditory pointing (and on some unpublished results by Bermant which are not available for discussion). Canon found, in two conditions where subjects had been instructed to point, respectively, at the visual and at the auditory target during exposure, that, after exposure to discordant visual and auditory information, pointing at the sole auditory target was shifted in the direction of the previous visual target. For the visual pointing condition, the suggested mechanism is that, when attempting to point at the visual target, the subjects were necessarily pointing to the side of the auditory target, and that they persisted doing that when instructed to point at the sole auditory target. In the condition where the instructions during exposure to conflict were to point at the auditory target, the subjects would similarly have gone on pointing to the side of the target, after doing it during the exposure trials. Here, however, an additional principle is needed to account for displaced pointing during the exposure phase. The authors suggest a sort of cue substitution mechanism: because visual localization is more accurate than auditory localization, the subjects "choose" to point to the visual target when it is available.

The assumption that subjects who were asked to point at a target would point to the side of its apparent location just because they had had to do it before, under a different set of instructions, sounds unlikely on common sense grounds. It has, in fact, now been rejected experimentally for the case of a visuo-proprioceptive discrepancy by Kelso, Cook, Olson, and Epstein (1975), in a paper published after Choe et al.'s.

The main difficulty for the response learning assumption, however, comes from the experiments where aftereffects were produced at the level of a response which had not been performed during the conflict exposure period. Radeau and Bertelson (1973) have shown that simply monitoring synchronous, but spatially discordant, noise and light pulses for occasional reductions in intensity led to shifts in both visual and auditory pointing. Radeau (1973), using the same procedure, has produced shifts in both hand pointings and settings of visual and auditory straight-ahead. These results can obviously be better accommodated by the assumption of a recalibration of the function relating apparent position to sensory data than by the response learning notion.

One can disagree with the extreme suggestion made by Choe et al. without automatically denying any intervention of postperceptual factors. There is little doubt that conscious inference may have played a role in producing some of the ventriloquist effects 
reported in the literature, especially in those studies where realistic familiar situations were presented or simulated. For instance, when Jackson (1953) asked his subjects to say which of several steam kettle whistles was producing a sound, when they could see steam coming from one of the whistles, the subjects probably based their responses on their knowledge of the principle of steam whistles more than on sensory evidence regarding sound location. A similar mechanism may have been at play in the extreme cases of ventriloquism reported for the subjects who went about their daily activities wearing devices which produced a left-right reversal of the visual (Stratton, 1897) or of the auditory input (Wiley, Inglis, \& Pearce, 1.937; Young, 1928). It would certainly be useful to know more about the respective contributions of perceptual and postperceptual adjustments. Does, for instance, ventriloquism produce the same effects when it is strongly facilitated by instructions or by contextual cues, as in the above realistic situations, and when it is based only on analysis of the pattern of 'auditory and visual input? It must, however, be realized that the separation is not an easy one to operate, for there is no sharp boundary between perceptual and postperceptual inference processes, as is shown, for instance, by the many examples where verbal knowledge facilitates or inhibits the formation of a percept (e.g., Leeper, 1935).

\section{Ventriloquism as Auditory-Visual \\ Interaction or Visual Dominance}

In their title, and at several places in the paper, the authors equate auditory-visual sensory interaction with visual dominance. In so doing, they follow a long tradition where ventriloquism has been defined as the effect of vision on auditory localization (e.g., Jackson, 1953; Thomas, 1941; Weerts \& Thurlow, 1971; Welch, 1974). This assimilation is nevertheless not acceptable, for there are many demonstrations of the opposite influence. Canon (1970), whom the authors quote, but only for the demonstration of auditory aftereffects, has also found visual aftereffect of exposure to audio-visual conflict. Visual aftereffects were first reported by Radeau and Bertelson (1969) and found again in several subsequent papers (Radeau, 1973, 1974; Radeau \& Bertelson, 1974). At the level of the immediate reaction, an attraction of visual pointing by conflicting auditory signals has been reported by Radeau (1974) and by Bertelson and Radeau (Note 1). The only good example of pure visual dominance is the result of Pick, Warren, and Hay (1969). So, although the picture is not as clear as could be wished, ${ }^{3}$ mutual attraction is a more plausible description of ventriloquism than visual dominance. It is interesting to note that, in two of the three early studies which Choe et al. quote as having demonstrated visual dominance (Jackson, 1953; Thomas, 1941), the subjects had only to match auditory and visual sources, so that nothing could possibly be inferred regarding the direction of dominance. The same argument applies to the experiment conducted by Choe et al., which also dealt with matching responses.

\section{REFERENCE NOTE}

1. Berteison, P., \& Radeau, M. The effect of structurization of the visual field on dominance in auditory-visual conflict. Paper presented at the 15th Annual Meeting of the Psychonomic Society. Boston, 1974.

\section{REFERENCES}

CANON. L. K. Intermodality inconsistency of input and directed attention as determinants of the nature of adaptation. Journal of Experimental Psychology, 1970, 84, 141-147.

CANON, L. K. Directed attention and maladaptive "adaptation" to displacement of the visual field. Joumal of Experimental Psychology, 1971. 88, 403.408.

Choe, C. S., Welch, R. B., Gilford, R. M., \& Juola, J. F. The "ventriloquist effect": Visual dominance or response bias? Perception \& Psychophysics, 1975, 18, 55-60.

JACXson. C. V. Visual factors in auditory localization. The Quarterly Journal of Experimental Psychology, 1953. 5, $52 \cdot 65$.

Kelso, J. A. S., Cook, E., Olson, M. E., \& Epstein, W. Allocation of attention and the locus of adaptation to displaced vision. Joumal of Experimental Psychology: Human Perception and Performance, 1975, 1. 237-245.

LEEPER, R. A study of a neglected portion of the field of learning - the development of sensory organization. Journal of Genetic Psychology, 1935. 46, 41-75.

Pick, H. L.. JR., Warren, D. H., \& Hay, J. C. Sensoty conflict in judgments of spatial direction. Perception \& Psychophysics, 1969, 6. 203-205.

Radeau. $M$. Adaptation au déplacement prismatique sur la base d'une discordance entre la vision et l'audition. L'Année Psychologique, 1974, 74, 23-34.

Radeau. M.. \& Bertelson, P. Adaptation à un déplacement prismatique sur la base de stimulations exafférentes en conflit. Psychologica Belgica, 1969, 9, 133-140.

Radeau, M.. \& Bertelson. P. The aftereffects of ventriloquism. The Quarterly Joumal of Experimental Psychology. 1974. 26. 63-71.

Stratton, G. M. Vision without inversion of the retinal image. Psychological Review, 1897, 4, 341-360, 363-481.

Thомаs, $G$. J. Experimental study of the influence of vision on sound localization. Joumal of Experimental Psycholog. 1941. 28. 167.177.

Thurlow. W. R. G., \& Jack, C. E. Certain determinants of the "ventriloquism effect." Perceptual and Motor Skills, 1973, 36, 1171-1184.

Warren, D. H. \& Cleaves. W. T. Visual-proprioceptive interaction under large amounts of conflict. Journal of Experimental Psychology. 1971, 90, 206-214.

Weerts. T. C.. \& Thurlow. W. R. The effect of eye position and expectation on sound localization. Perception \& Psychophysics, 1971, 9. 35-39.

WFLCH. R. B. Research on adaptation to rearranged vision: 1966-1974. Perception. 1974. 3. 367-392.

Wiley, C. F., Inglis, E. \& Pearce, C. H. Reversal of 
auditory localization. Journal of Experimental Psychology, 1937, 20, 114-130.

YouNG, P. T. Auditory localization with acoustical transposition of the ears. Journal of Experimental Psychology, 1928, 11, $399-429$.

\section{NOTES}

1. A reduction of $\mathrm{d}^{\prime}$ can also result from an increase in the variance of $x$ with no shift of the means. There is, however, no reasonable basis on which to predict this kind of effect for sensory interaction.
2. The assumption that recalibration occurs only when apparent fusion is experienced is disputable. Some mutual attraction between proprioceptive and visual data can still occur with large discrepancies, such as $60^{\circ}$, with which fusion is impossible (Warren \& Cleaves, 1971).

3. Bertelson and Radeau (Note 1) have found that discordant auditory signals affect visual localization when the visual input is reduced to a single luminous point in an otherwise dark field, not with a structured background.

(Received for publication December 26, 1975, revision accepted March 25, 1976.) 